sachen wird bekanntlich eine Mischung von konzentrierter Schwefelsäure und Kaliunı bichromat-Lösung enıpfohlen, doch auch bei Verwendung dieser Flüssigkeit konnte ich nicht eine viel weitergehende Zer'störung der Tuberkelbazillenleiber als bei dem Gebrauch konzentrierter Sclıwefelsäure allein bemerkeı. Die Hauptsache bei del Reinigung - besonders del Objektträger - besteht wohl vielmehr in einel gründ lichen nllecllanisclien Entfernung der fixierten Massen. Daß dies nicht ganz leicht ist, möge nicht verschwiegen werden, unı so mehr ist die Beachtung dieser Regel geboten.

Dasselbe, was von den zur Untersuchungsmethodik zu verwendenden Glassachen gilt, trifft selbstverständlich auch von den Gefäßen zu, in dellen die Patienten Sputum, Urin etc. zur Untersucliung einsenden. Hier würden natürlich nur sterile Gläser $z u$ verwenden sein, ebenso wie bei der Untersuchung und Aureicherung selbst nur sterile Kolben, Zentrifugengläser etc. in Benutzung zu kommen haben.

Wenn wir nun durch Beachtung des eben Erwähnten diese Fehlerquelle ausschließen, so müssen wir uns ferner bei der mikroskopischen Diagnose vergegenwärtigen, daß diese nur auf ,säurefeste Stäbchen" gerichtet ist, respektive auf stäbchenartige Gebilde, die die Eigenschaft haben, Fuchsin schwer und nur durch Wärme aufzunehmen und den Farbstoff bei nachträglicher Behandlung niit Säuren festzuhalten. Wir müssen da-

\section{Ueber die Verhütung der mikroskopischen Fehldiagnose der Tuberkelbazillen.}

Von Dr. Hans Bontemps in Hamburg-Altona.

Die in neuerer Zeit moderiı gewordenell Anreicherungsmethoden zum Zwecke des Nachweises von Tuberkelbazillen in Sputum, Urin, Stuhl, Blut etc. haben ergeben, daß wir jetzt in sehr vielen Fällen schon auf mikroskopischem Wege zu einer positiven Diagnose gelangen, in denen wir in dell Originalausstrichen vergeblich nach Tuberkelbazillen suchten. Da wir in diesen Fällen früher immer den Tiel- und Kulturversuch nötig gehabt hätten, um zu eiller positiven Diagnose zu gelangen, so drängt sich die Frage auf, ob wir uns nicht jetzt bei sachgemäßer Anwendung z. B. der Uhlenhuthschen Antiforminanreicherungsmethode - mit dem Ausfall der mik.roskopischen Untersuchung allein zufrieden geben können. Hier muß man, unter der Voraussetzung, daß die Technik und Sorgfalt des Untersuchers den weitgehendsten Ansprïchen genügt, zugeben, daß in der Mehrzahl der Fälle wohl auf den Tier- und Kulturversuch verzichtet werden kann. Lassen sich ill einer genügend großen Menge des zu untersuchenden Materials nach vorgenommener Anreicherung und gründlichem Auszentrifugieren nikroskopisch keine säurefesten Stäbchen findell, so kann man sich mit dem negativen Ausfall der Untersuchung als zu Recht bestehend zufrieden geben.

Anders liegen die Verhältnisse jedoch bisweilen in positiven Fällen; wollen wir daher danach trachten, uns mit der nikroskopischen Untersuchung allein zu begnügen, so erscheint es mir wünschenswert, einmal denjenigen Gebilden nachzuspüren, díe uns mikroskopisch Tuberkelbazillen vortäuschen können.

Ich habe mir daher in diesen Ausführungen die Aufgabe gestellt, auf Fehlerquellen verschiedener Art hinzuweisen, durch deren Nichtachtung gelegentlich Fehldiagnosen entstehen können; daher möchte ich nicht versäumell, auch das Nachstehende mit anzuführen.

Alle zur Untersuchung benötigten Glassachen mïssen, da aus ökonomischen Gründen eine nur einnalige Verwendung ausgeschlossen ist, aufs Sorgfältigste nach jedesmaligem Gebrauch gereinigt werden. Einfaches Auskochell in Sodalösung genügt meist nicht, da die Tuberkelbazillen besonders all Objektträgern und in den Kuppen der Zentrifugengläser festhaften; ihrer Vernichtung ist daher eine besondere Sorgfalt zuzuwenden. Dasselbe gilt auch von den ïbrigen Glassachen, die man bei den Anreicherungsmethoden benötigt. Ich konnte beobachten, daß gefärbte Tuberkelbazillen selbst in kollzentrierter Sch wefelsäure längere Zeit weder morphologisch, noch tinktoriell beeinflußt wurden und daß sie, selbst wenn sie die Farbe einbüßten, bei einer nachträglichen Färbung nach Ziehl-Neelse $\mathbf{n}$ wieder gut erkennbar wurden.

Zur Reinigung der mit Tuberkelbazillen behafteten Glasher unsere Diagnose auf dieser Eilıschränkung aufbauen und wissell, was für andersartige Mikroorganismen und sonstige säurefeste Substanzen uns eine Fehldiagnose vortäuschen können.

Da'wären denn in erster Linie die verscliedenen Arten der säurefesten Stäbclien zu nennen, welche biologisch den Tuberkelbazillell nahestehen. Im Bakterienreich ist es die Gruppe der Mycobakterien. Hierzu gehören (außer den Tuberkelbazillen) die Smegmabazillen und die in Milch, Butter, Wasser, Gras, Mist etc. vorkommenden verschiedenell Arten.

Um Verwechslungen dieser im allgemeinen harmlosen, saprophytisch vorkommenden Arten mit Tuberkelbazillen auszuschließen, ist daher zu beachten, daß die zu untersuchenden Materialien nicht nachträglich verunreinigt werden. Unı Smegmabazillen auszuschließen, sollte daher Urin - besonders beim weiblichen Geschlecht - nur durch Katheter entnomnen und in sterilen Gefäßen aufgefangen werdelı. Um andere säurefeste Stäbchenartell nicht in das Untersuchungsmaterial hineinzubringen, sollte bei den Anreicherungsmethoden nur steriles destilliertes Wasser in Anwendung konımen.

Bei Befolgung dieser Maß3regeln ist man dann sicher, daß keine alldersartigen säurefesten Stäbchen das Untersuchungsmaterial infiziert haben. Handelt es sich nun um den Nachweis von Tuberkelbazillen in Milch, Butter, Wasser etc., also in Medien, in denen säurefeste Stäbchen saprophytischer Art vorkommen, so können wir, falls die mikroskopische Betrachtullg ein positives Resultat ergibt, zur sicheren Diagnostik den Tierund Kulturversuch nicht entbehren.

AuBer den erwähnten, zur Gruppe der Mycobakteriell gehörigen Stäbchen gibt es dann noch gewisse Aktin omycesarten, die sich im bakteriologischen Sinne säurefest verhalten. Da die Aktinomyceten jedoch durch Größe und Gestalt sich leicht von den Tuberkelbazillen unterscheiden lassen, so wird ihre Erkennung in allgemeinen kaum auf Schwierigkeiten stoßen.

Nun gibt es ferner aber noch Substanzen nicht bakterieller Art, die im bakteriologischen Sinne als säurefest zu bezeichnen wären, und über diese ist, soweit ich in der Literatur in Erfahrung bringen konnte, bislang wenig berichtet.

Ueber eine Verwechslung dieser Art, die mir selbst begegnet ist und die verdient, bekannt zu werden, möchte ich im folgenden berichtell:

Vor einigen Monaten bekain ich ein Sputum zur mikroskopischen Untersuchung eingesandt. Iml Originalausstrich fanden sich, nach Zie hl. Neclsen grä̈rbt, keine säurefesten Stäbchen; dagegen kieBen die Präparate, die nach der Uhlenhuthschen Antiforminanreicherungsmethode hergestellt und nach Ziehl-Neelsen gefärbt waren, vereinzelte säurefeste Stäbchen erkennen; diese erschienen allerdings etwas dicker, als Tuberkelbazillen für gewöhnlich zu sein pflegen, auch ließen sich Andeutungen von Verzweigungen konstatieren; immerhin erinnerten sic 
durchaus an Formen von Tuberkelbazillen, wie sie besonders für den Typus bovinus charakteristisch sind. Ich benachrichtigte daher den behandelnden Arzt von dem mikroskopisch erhaltenen Resultat im positiven Sinne. Da ich auf meine Mitteilung hin von dem Kollegen erfuhr, daß dies Sputum bereits mehrfach mit negativem Erfolge 1nntersucht wäre, so bat ich zur Klärung dieses Falles um nochmalige Finsendung, um der mikroskopischen Untersuchung den Tier- und Kulturversuch mit anzuschließen.

In dem nach einigen Tagen erhaltenen Sputum fanden sich mikroskopisch wiederum die gleichen sälurefesten Stäbchenformen, und zwar fand ich auch diesmal diese erst in den Präparaten, die nach der Antiforminanreicherıngsmethode hergestellt waren; in den Originalansstrichen waren sie nicht auffindbar gewesen. Da die beiden geimpften Meerschweinchen einige Tage nach der Impfung an Pnelımonie eingingen und mir daher keine Diagnose ergaben, so war auch diesmal das Resultat ebenso zweifelhaft.

In einem weiter erbetenen Sputum fand ich nun, und zwar diesmal schon in den Originalausstrichen, zahlreiche säurefeste Stäbchen, teils ohne, teils mit Verzweignngen; des weiteren aber fanden sich neben diesen alle möglichen säurefesten Gebilde, weit verzweigte Gabelformen und ganze Netzwerke säurefester Substanz (s. Fig. 1). Schließlich sah ich auch zienlich große, dreieckige, pyramidenartige Körperchen, die aus einem waben- oder netzartigen Gerüst säurefester Substanz bestanden die mit Affektionen der Luftwege behaftet sind - wie sich dies auch in dem beschriebenen Falle ergab - Lycopodium als Pillenstreupulver mit einnehmen, wodurch eine Verunreinigung des Sputums mit Lycopodium herbeigeführt werden kann, so dürfte es sich empfehlen, vor Sputumuntersuchungen niemals Pillen zu verordnen. Da Lycopodium ferner außerordentlich leicht aspiriert werden kann, so denke man auch an die säurefeste Eigenschaft und die Täuschung, die daraus resultieren kann, wenn es sich darum handelt, Sputa von Apothekern und Drogisten zu untersuchen, die erwiesenermaßen sehr häufig Lycopodium in ihren Luftwegen beherbergen.

Vergeblich hatte ich in der Literatur nachgesucht, ob ich diese Eigenschaft des L ycopodiums bereits beschrieben fände: ich konnte jedoch nichts darüber entdecken. Gelegentlich einer zufälligen Unterhaltung, die ich mit dem Kollegen Dr. Delban co (Hamburg) hatte, erfuhr ich vol ihm, dab er bereits vor neun Jahren in ähnlicher Weise wie ich durch das Vorhandensein von Lycopodium im Sputum auf diese Eigenschaft der Bärlappsamenspore gestoßen ist und sie 1903 zum Gegenstand einer Veröffentlichung in den Monatsheften fïr praktische Dermatologie gemacht hat. - Da es sich jedoch in diesem Falle um ein in einer Pillenschachtel eingesandtes Sputum gehandelt hatte, so waren Lycopodiumsporen sehr zahlreich darin enthalten. Delbanco hatte daher in jedem Gesichtsfelde außer den Stäbchen auch die übrigen, weniger stark zerstiickelten, Teilchen gesehen; hierdurch war er sogleich darauf aufmerksam gemacht, daß die säurefesten Gebilde nichts mit Tuberkelbazillen zı tun haben könnten. Sind jedoch. Lycopodiumsporen nur in mäBiger Anzahl im Sputum vorhanden, wie dies nach dem Gebrauch von Pillen ja nur $z u$ sein pflegt, und kommt dann ferner noch die Anreicherungsmethode hinzu, durch welche die Sporen in kleine stäbchenartige Gebilde zerlegt werden, so ist es sehr wohl inöglich, daß im mikroskopischen Bilde sich den Tuberkelbazillen ganz ähnliche Stäbchen finden; jedenfalls läßt sich die $\mathrm{Ab}$ wesenheit von Tuberkelbazillen in diesem Fall nicht ausschlieBen. Derselben Ansicht verleiht auch Dolbanco in seiner Stäbchenartige Teilchen von zertrümmerten
Lycopodiumsporen. Lycopodiumsporen. und die sich erst bei langsamem Hin- und Herdrehen der Mikrometerschraube infolge ilirer Größe als die geschilderten Gebilde dokumentierten, während sie zunächst Farbstoffklumpen vorgetäuscht hatten (s. Fig. 2), (wie man sie bisweilen in nicht genïgend entfärbten Ziehlpräparaten sieht).

Die genaue Dmchsicht der Präparate ließ erkennen, daß die ge sehenen säırefesten Stäbchenformen zweifellos nicht Tuberkelbazillen waren und daß sie, wie auch die übrigen säurefesten Gebilde, die Gabeln, die Netze etc. Zerfallsprodukte der dreieckigen, pyramidenartigen Körperchen waren. Es ging dies daraus hervor, daß einzelne der Stäbchen noch in direktein Zusammenhang mit den pyramidenartigen Körperchen standen, während alle möglichen Uebergangsformen in der Ungebung sichtbar waren.

Was stellten diese Körper nun dar? Unbekannt waren sie mir nicht, und ich erinnerte mich, sie schon bei anderer Gelegenheit gesehen zu haben. Auch verschiedene Kollegen, denen ich den Befund gezeigt hatte, wuBten nicht, um was es sich handelte. Durch Zufall kamen wir jedoch bald auf das Richtige; mein Kollege, Dr. Schröder (jetzt Assistent am Merseburger städtischen Krankenhaus), mikroskopierte verschiedene pflanzliche Pulver seiner pharmakognostischen Sammlung und fand hierbei die ihm von mir gezeigten fraglichen Gebilde identisch mit Lycopodiumsporen.

Alle ferneren Prüfungen bestätigten die Identität dann auch; es erwies sich, daß Lycopodium, nach Ziehl-Neelsen gefärbt, als säurefest zu bezeichnen ist und daß es besonders bei dem Antiforminanreicherumgsverfahren zu einem Zerfall der Sporen kommt. Hieidurch werden dam Formen vorgetäuscht, die bei nachträglicher Ziehl-Neelsenfärbung Tuberkelbazillen außerordentlich ähnlich sehen.

Da nun das L y copodiu n, ein so gebräuchlicher pharmakologischer Artikel, speziell gerade als Streupulver für Pillen in Betrasht kommt und da die Möglichkeit besteht, daß Patienten,
Arbeit, die im übrigen auch die Säurefestigkeit der Korkzelle betrifft, berechtigten Ausdruck. - In solchen Fällen, wo sich Lycopodiun im Sputum findet, können wir dann also den Tier- und Kulturversuch nicht entbehren.

Trotzdem Delban co bereits 1903 auf das Erwähnte hingewiesen hat, glaubte ich dennoch es nicht unterlassen zu dürfen, dies nochmals in einer allgemeinen medizinischen Zeitschrift zu tun, da ich überzeugt bin, daß nicht nur mancher Praktiker sondern auch mancher Mikroskopiker und Bakteriologe aus Unkenntnis der säurefesten Eigenschaft der Lycopodiumspore gelegentlich vor einem Rätsel steht, sofern er nicht eine Fehldiagnose stellt.

Interessant wäre es nun zu erfahren, ob es noch andere Substanzen gibt, die sich als säurefest erweisen. Außer der Lycopodiumspore und den Korkzellen habe ich diese Figenschaft noch bei dem im Scheibenhonig vorkommenden Wabenwachs feststellen können. Wahrscheinlich gibt es auch noch sonst Substanzen, dic sich ähnlich verhalten; die Feststellung und Publizierung derselben wäre jedenfalls sehr erwïnscht. besonders in Fällen, die zu Verwcchslumgen führen können. 